\title{
Los animales como seres vivos dotados de sensibilidad ante el interés común en la ganadería nicaragüense y los acuerdos de la OIE y el OIRSA
}

\author{
Alberto Argüello Sacasa \\ Máster en Derecho Animal \& Sociedad \\ Corresponsal del ICALP para Centroamérica
}

Recepción: Abril 2018

Aceptación: Junio 2018

\section{Resumen}

Este ensayo tiene como fin dar a conocer la importancia de la "sentiencia animal" en la Región Centroamericana, ante los acuerdos de la Organización Mundial de Sanidad Animal (OIE) y el Organismo Internacional Regional de Sanidad Agropecuaria, (OIRSA), tomando como referencia la Constitución Política y las Normas Técnicas Obligatorias del país de Nicaragua, enfocándonos en la inducción de síntomas de estrés por calor en el ganado, como un agravante que desafía el cambio climático en el sector ganadero nicaragüense.

Palabras clave: seres vivos dotados de sensibilidad, OIE, OIRSA, cambio climático, bien común

Abstract. Animals as living beings endowed with sensibility, the common interest in Nicaraguan livestock sector, and the OIE and OIRSA agreements

This essay aims to raise awareness of the importance of "Animal Sentience" in the Central American Region, before the agreements of the World Organization for Animal Health (OIE) and the International Regional Organization for Animal Health (OIRSA), using as reference the Political Constitution and Obligatory Technical Standards of Nicaragua, focusing on the induction of heat stress as an indicator of symptoms in the livestock, as an aggravating factor for the climate change in the Nicaraguan cattle farming sector.

Keywords: Animal law, mistreatment, crime, dogs, cats, serial killer, animal conscience. 


\section{SUMARIO}

Introducción

1.La ganadería en el sistema agropecuario

2.Comportamiento de consumo centroamericano

3.Los acuerdos de la OIE y el OIRSA en la región centroamericana

4.La sentiencia animal ante el interés común en Nicaragua desde la Constitución política

5.El “estrés” causado por calor como un indicador de alerta para el bienestar animal ante el cambio climático

Conclusión

\section{INTRODUCCIÓN}

No podemos negar que, ante la constante interacción de los seres humanos y animales, se ha despertado una mayor sensibilidad global hacia una nueva forma de tratos más dignos para los animales. A nivel global, se viene abordando la importancia de la ética y buenas prácticas que deben adoptar los países referentes al bienestar animal. Hoy también nos enfrentamos a nuevas terminologías, derechos y declaraciones internacionales como son los estándares de bienestar animal delineados por la Organización Mundial de Sanidad Animal (OIE), como nuevas formas de tratos al utilizar a los animales de producción para consumo humano y lograr una inserción de los animales domésticos en la sociedad de forma más digna. Hoy por hoy, los consumidores exigen más información sobre el origen de sus alimentos; igual las formas de producirlos importan mucho al consumidor.

Es por ello que los consumidores reclaman más controles de calidad y certificados de sostenibilidad bajo sellos de tratos amigables, como una exigencia a la industria, sin importar su tamaño, sean grandes, medianas o micros. Hoy por hoy, todas enfrentan la misma realidad para prevenir el maltrato.

Tales exigencias nos obligan a examinar y escudriñar nuestras legislaciones país por país, para poder formular nuevas políticas, para garantía de los consumidores e interés común de la sociedad, y ante ese interés general, se debe reformular y redirigir una nueva ruta por el bien común, para promover nuevos espacios e incentivar más políticas de cambio en nuestras bases sociales como región centroamericana.

En este sentido, es necesario formular un nuevo camino a las exigencias sociales en materia de bienestar animal, en la cultura, la pesca, el transporte, el mercado interior y exterior, la investigación, el desarrollo tecnológico, la religión y el espacio, ante una perspectiva universal de bienestar e interés social, para comenzar una nueva disposición de cambio en toda la matriz jurídica actual, promoviendo tratos más humanitarios hacia los animales para lograr así, un desarrollo sostenible por una sola salud.

\section{LA GANADERÍA EN EL SISTEMA AGROPECUARIO}

El origen etimológico del término ganado ${ }^{1}$, se define para aquellos animales que están sujetos a crianza o explotación. El término tiene estrecha relación con la era del "feudalismo", con sus riquezas y conexiones, con los términos de "pecunio ${ }^{2}$ ”, adjetivo que

\footnotetext{
${ }^{1}$ Ganado: “conjunto de animales sujetos a crianza o explotación”. Término de origen germánico. http://etimologias.dechile.net/?ganado

${ }^{2}$ Pecunio. https://dialnet.unirioja.es/descarga/articulo/1281667.pdf 
se refiere a la palabra rebaño o ganado como res o cabeza de ganado lanar, término perteneciente al dinero en efectivo. En latín significaba primitivamente "riqueza ganadera”, cuando la riqueza se valoraba según la cantidad de reses que se poseía.

El término "ganado" se origina del verbo *ganar, ${ }^{*}$ codiciar, *desear, de la evolución semántica de ganar, que habría estado condicionado con el influjo de la lengua germánica

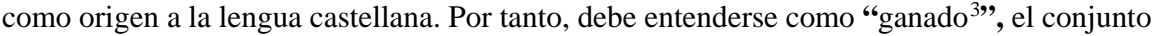
de animales cuya crianza está destinada al consumo humano y debemos tener en cuenta que del ganado se obtienen una gran cantidad de alimentos tales como la carne, la leche y derivados, y bajo esta acción de ganar también surgen otras materias primas como el cuero, la lana y el estiércol. El término de ganar en la ganadería, se le atribuye por formar parte de un sector primario en la economía, que también tiene una relación directa con la industria textil, la industria alimenticia, industria peletera, la tauromaquia y las granjas escuelas.

Desde una perspectiva científica, la zootecnia es la disciplina que se centra en todo lo relativo a la producción de animales y la Medicina Veterinaria ${ }^{4}$ es la ciencia que se ocupa de la prevención, el diagnóstico y tratamiento de enfermedades en los animales y hoy en día, en esta nueva era, contamos con el Derecho Animal, que estudia todas las regulaciones nacionales e internacionales que vinculen a los animales de producción, que estudia el comportamiento de la sociedad en cuanto al consumo, protección y bienestar de los animales, tomando como punto de partida el reconocimiento a los animales como Seres Vivos dotados de Sensibilidad (Sentient Beings), nuevo enfoque que nos sirve para interrogar a las normas de derecho común vigente.

\section{COMPORTAMIENTO DE CONSUMO CENTROAMERICANO}

Centroamérica, políticamente, se divide en siete países independientes: Guatemala, Belice, Honduras, El Salvador, Nicaragua, Costa Rica y Panamá. Entre estos siete países, según la firma Kantar Worldpanel $^{5}$, expertos en comportamiento del mercado del consumidor en la región que dan seguimiento continuo al comportamiento de compra de hogares centroamericanos de manera quincenal, indican que debido al crecimiento interno bruto de los países, en Nicaragua ${ }^{6}$ y Panamá el consumo en el 2017 creció considerablemente, resaltando que la canasta de consumo masivo, que incluye productos alimenticios de vida, lácteos, de cuidado personal y del cuidado del hogar, siguen en aumento. También en el mes de septiembre del $2017^{7}$ la Secretaría Ejecutiva del Consejo Monetario Centroamericano (Secmca) indicó que la inflación centroamericana se mantuvo en niveles históricamente bajos en ambos países. Curiosamente, según datos de “CentralAmericanData.com”, el consumo anual per cápita de carne de pollo ${ }^{8}$ en los países centroamericanos aumentó de 19,8 kilos en 2008 a 25,9 kilos en 2016, y el crecimiento fue

\footnotetext{
${ }^{3}$ Definición ABC. Ganado concepto. https://www.definicionabc.com/economia/ganado.php

${ }^{4}$ Real Academia Española: Veterinario, ria. http://dle.rae.es/?id=bi2iFp6

${ }^{5}$ Kantar World Panel es un experto global en el comportamiento del consumidor. A través de un continuo monitoreo, avanzados análisis y soluciones a la medida, Kantar World Panel inspira decisiones para marcas, distribuidores, analistas de mercado y organizaciones públicas en todo el mundo. www.kantarworldpanel.com

6 http://www.elnuevodiario.com.ni/economia/420834-nicaragua-panama-lideran-crecimientoconsumo-ca/

${ }^{7}$ http://www.elnuevodiario.com.ni/economia/441092-bajos-precios-alimentos-nicaragua-panama/

8 Central América Data. Desde el 2007 trabaja en la información relevante para la toma de decisiones de negocios en Centroamérica para brindarla con visión regional a los tomadores de esas decisiones. El portal es líder en la publicación de análisis y noticias regionales más relevantes y constituye la mejor y más amplia fuente de información sobre economía y negocios de América Central. Véase, Crece Consumo de Pollo en Centroamérica (5/10/2017) disponible en

https://www.centralamericadata.com/es/article/home/Crece_el_consumo_de_carne_de_pollo_en_Centr oamrica
} 
impulsado principalmente por Panamá. De igual forma, la carne porcina 9 en los países de la región aumentó de 4,6 kilos en 2012 a 5,3 kilos en 2016 y el crecimiento fue impulsado principalmente por Panamá y Costa Rica y, aunque la carne bovina no ha tenido sus mejores cifras comparándose a los años 2015 y 2016, los países centroamericanos no dejan de exportar, entre ellos Nicaragua con \$421 millones, seguido de Costa Rica con \$62 millones, Panamá con \$16 millones, Guatemala con \$5 millones y Honduras con \$3 millones y los destinos han sido los países de EE.UU., 16\% Venezuela, 8\% Puerto Rico, 5\% México y 5\% Taiwán, según el portal de Central América Data, lo que indica que los animales seguirán siendo una prioridad para el consumo humano.

\section{LOS ACUERDOS DE LA OIE Y EL OIRSA EN LA REGIÓN CENTROAMERICANA}

Entre el año 2005 y el 2009 se adoptaron en la región Centroamericana dos acuerdos importantes que determinarían el futuro de la ganadería en la región, el primero fue el 21 de mayo del año 2005 ante la Organización Mundial De Sanidad Animal ${ }^{10}$ (OIE), considerando el desarrollo de intercambio de animales y sus productos y la creación de un entorno legislativo apropiado mediante el establecimiento de "normas aceptables" por todos los actores implicados, teniendo como principal objetivo por parte de la OIE facilitar el comercio internacional de animales y el de sus productos, mediante la elaboración de normas sanitarias internacionales, considerando el mandato de la OIE, en materia de seguridad sanitaria de los alimentos y el bienestar de los animales. El acuerdo en el artículo $2^{\circ 11}$ estableció que tanto la OIE como el Organismo Internacional Regional de Sanidad Agropecuaria (OIRSA) ${ }^{12}$ ayudan y cooperan en función de alcanzar una "Armonización de Legislaciones y Reglamentaciones Sanitarias de Animales y Zoonosis”. El segundo ${ }^{13}$ acuerdo fue adoptado el 30 de abril del año 2009. Éste acuerdo de Cooperación, entre la Secretaría General del Sistema de la Integración Centroamericana (SG-SICA) y el Organismo Internacional

\footnotetext{
${ }^{9}$ Central América Data. Título: Centroamérica consume más carne de cerdo. En el último quinquenio el consumo anual per cápita de carne porcina en los países de la región aumentó desde 4,6 kilos en 2012 a 5,3 kilos en 2016, y el crecimiento fue impulsado principalmente por Panamá y Costa Rica. https://www.centralamericadata.com/es/article/home/Centroamrica_consume_ms_carne_de_cerdo

10 Acuerdo entre el Organismo Internacional Regional de Sanidad Agropecuaria (OIRSA) y La Organización Mundial De Sanidad Animal (OIE): http://www.oie.int/es/quienes-somos/textosprincipales/acuerdos-de-cooperacion/acuerdo-entre-el-organismo-internacional-regional-de-sanidadagropecuaria-y-la-organizacion-mundial-de-sanidad-animal/

11 Acuerdo entre el Organismo Internacional Regional de Sanidad Agropecuaria (OIRSA) y la Organización Mundial De Sanidad Animal (OIE) adoptado el 21 de mayo de 2005. Artículo 2: La OIE y el OIRSA cooperarán en los ámbitos siguientes, sin que esta lista sea excluyente de otras actividades que ayuden a alcanzar sus objetivos: Armonización de legislaciones y reglamentaciones sanitarias animales y las zoonosis; Cooperación técnica en el ámbito de la salud animal y zoonosis; Intercambio de información científica, difusión de publicaciones y programas de trabajo; Fortalecimiento de los sistemas de vigilancia epidemiológica en la región; Difusión del sistema de información sanitaria de la OIE sobre la ocurrencia de enfermedades animales y zoonosis; Organización de consultas, seminarios, cursos y jornadas de capacitación tanto para los Servicios Veterinarios Oficiales, como sector Privado; Organización de consultas y seminarios sobre actualización y modificación de los estándares de la OIE; Difusión y promoción de elaboración de propuestas y análisis y actualización de las normas y lineamientos de la OIE; Difusión y promoción de la aplicación de los Códigos y Manuales Terrestres y Acuáticos de la OIE; Refuerzo de los Servicios Veterinarios, tomando en cuenta las normas de la OIE en su evaluación y calidad, mediante el apoyo para la organización de cursillos, auto-evaluación, herramientas para inversiones públicas y privadas y la formación en los establecimientos universitarios de vocación veterinaria y agroalimentaria.

12 https://www.oirsa.org/informacion.aspx?id=5

13 Acuerdo de Cooperación entre la Secretaría General del Sistema de la Integración Centroamericana (SG-SICA) y el Organismo Internacional Regional de Sanidad Agropecuaria (OIRSA). 30/4/2009, http://www.sica.int/consulta/documento.aspx?idn=35333\&idm=1
} 
Regional de Sanidad Agropecuaria OIRSA, se firmó con el objeto de crear condiciones favorables para la salud animal, para así facilitar el comercio de productos agropecuarios de la región, conforme a lo establecido en el artículo 1 del documento citado.

Cabe mencionar que el OIRSA ${ }^{14}$ se fundó hace 61 años y entre su roles está brindar cooperación técnica a los Ministerios, Secretarías de Agricultura y Ganadería por ser Estados miembros, así como protección y desarrollo de sus recursos agropecuarios, a fin de garantizar una producción alimentaria sana y segura para el bienestar de la población humana. En este aspecto, el organismo se acciona y enfoca en 4 áreas técnicas estratégicas:

1. Salud Animal

2. Sanidad Vegetal

3. Servicios Cuarentenarios

4. Inocuidad de los Alimentos

Si bien es cierto en materia de comercio que las cuatro áreas son vitales, los acuerdos mencionados deben ser retomados nuevamente para valorar el alcance de lo que generó la armonización normativa y las regulaciones en Salud Animal e Inocuidad de Alimentos de origen animal, ya que son necesarios para fortalecer y desarrollar los programas sanitarios regionales para las especies, los cuales ayudan a alcanzar una Armonización de Legislaciones y Reglamentaciones Sanitarias de Animales y Zoonosis.

Es de vital importancia resaltar que las acciones estratégicas del OIRSA formaron parte de la política internacional de la OIE para lograr alcanzar lo que hoy se conoce como "Una sola salud ${ }^{15 ”, ~ i n i c i a t i v a ~ q u e ~ s e ~ d e s a r r o l l o ́ ~ d e ~ l a ~ m a n o ~ c o n ~ l a ~ a r m o n i z a c i o ́ n ~ n o r m a t i v a ~}$ regional, y es precisamente en esta línea de acción, que hoy día, la ciencia jurídica del Derecho Animal, hace su interrogatorio sobre si se debe incluir la nueva consideración hacia los animales, hoy vista desde una postura científica, bajo un sorprendente giro que vino a dar la Declaración sobre la Consciencia ${ }^{16}$, manifiesto hecho bajo consenso para el público que no es científico el 7 de julio del año 2012, en la Universidad de Cambridge, por connotados científicos que expresan al mundo la situación sobre la consciencia de los animales humanos y no humanos, manifiesto que espero que sirva para incentivar tratos más humanitarios hacia los animales. Con esto quiero decir que hoy no basta con decir que únicamente los seres humanos son conscientes y capaces de experimentar dolor, sensaciones físicas, miedo, felicidad y percibir experiencias negativas y positivas, sino también los animales. El mundo debe reconocer que los animales son seres vivos conscientes y bajo este nuevo enfoque el Derecho Animal escudriña todas las legislaciones que vinculan a los animales de Nicaragua.

En este aspecto es necesario volver a valorar los acuerdos entre el OIRSA y la OIE, para que se promueva un cambio en la legislación de Nicaragua y sean cambios verdaderamente eficientes, oportunos y contundentes, donde todo este esfuerzo sea parte de un resultado y compromiso adquirido para mejorar la calidad de vida de las personas y animales.

Para retomar las líneas de acción del OIRSA y la política internacional de la OIE, tomando como referencia el país de Nicaragua por ser líder en exportaciones de carne bovina y el único país de la región que reformó su Constitución en el año 2014 reconociendo una protección muy especial a la madre tierra, ahora tenemos un reto mayor puesto que:

"Debemos proteger y restaurar la integridad de los ecosistemas, con especial

\footnotetext{
${ }^{14}$ https://www.oirsa.org/informacion.aspx?id=5

15 OIE “Una Sola Salud”. http://www.oie.int/es/para-los-periodistas/onehealth-es/

${ }^{16}$ La Declaración de Cambridge sobre la Conciencia (Cambridge Declaration on Consciousness) es un manifiesto firmado durante una serie de conferencias respecto a la conciencia en los animales humanos y no humanos, realizadas en julio de 2012, en la Universidad de Cambridge (Reino Unido). http://www.anima.org.ar/wp-content/uploads/2016/03/Declaración-de-Cambridge-sobre-la- 
preocupación por la diversidad biológica y por todos los procesos naturales que sustentan la vida. - La nación nicaragüense debe adoptar patrones de producción y consumo que garanticen la vitalidad y la integridad de la madre tierra, la equidad social en la humanidad, el consumo responsable y solidario y el bien vivir comunitario. (Art $60^{17} \mathrm{Cn}$.)

\section{LA SENTIENCIA ANIMAL ANTE EL INTERÉS COMÚN EN NICARAGUA DESDE LA CONSTITUCIÓN POLÍTICA}

Debemos partir de que uno de los principios rectores que ha desarrollado la OIE en la Unión Europea es el Bienestar Animal y bajo ese modelo estratégico de trato preferencial a los animales, se ha venido marcando una nueva ruta en la ciencia y educación de la UE, donde se ha incrementado un mayor nivel de conciencia y respeto a los animales para el consumo humano que hoy forman parte de la columna vertebral normativa, derivando grandes compromisos legislativos a cumplir por los países miembros de la unión.

Por tal motivo, vale la pena mencionar que la transferencia del modelo y adaptación de la política normativa internacional de la OIE fue adquirida por el OIRSA y el modelo causó un efecto muy significativo en la aprobación de leyes especiales de Bienestar Animal en varios países de la región y sobre todo en el país de Nicaragua. Los sistemas productivos de las explotaciones agropecuarias han sido uno de los beneficiados $\mathrm{y}$, muy a pesar de que todas las normas de bienestar en bovinos hacen referencia a la política de bienestar animal de la comunidad europea, la armonización regional no vislumbró el impacto y compromiso que debió generar la armonización en el resto de la legislación del derecho positivo de los países miembros de la región, o mejor dicho en el país de Nicaragua.

Ante lo expuesto, resalto que las referencias que tienen las normas técnicas obligatorias de Nicaragua que fueron armonizadas bajo el asesoramiento del OIRSA por acuerdo con la OIE, hoy día resaltan y expresan el compromiso que la UE adquirió bajo el Tratado de Funcionamiento de la Unión Europea (TFUE), que entró en vigor el 1 de diciembre del año 2009, que en su artículo $13^{18}$ es preciso al tratarse de formular y aplicar las políticas de la Unión en materia de agricultura, pesca, transporte, mercado interior, investigación, y desarrollo tecnológico y espacio; por lo que la Unión y los Estados miembros tendrán plenamente en cuenta las exigencias en materia de bienestar de los animales como "seres sensibles ${ }^{19}$ ".

Sobre la dimensión de la ruta jurídica expuesta, es necesario analizar si los acuerdos de la OIE y el OIRSA han tenido un impacto positivo en materia de bienestar animal en el país de Nicaragua y, ¿por qué no?, valorarlo inclusive en la región centroamericana, o bien, si la OIE y el OIRSA deben replantearse una nueva estrategia para que la política de estado en cada país considere con prioridad volver a retomar una revisión sobre el reconocimiento a los animales como seres vivos dotados de sensibilidad como política de estado, y promueva así un verdadero cambio significativo. Si bien es cierto se han dado cambios sustanciales, los resultados no han sido los más oportunos (al menos en Nicaragua), puesto que se sigue apreciando una marcada indiferencia y apatía hacia los temas de bienestar animal.

4.1 El Bienestar Bovino del país de Nicaragua ante una Esterilidad Normativa

La revista Forbes México ${ }^{20}$, versión para Centroamérica, se refiere al país de

17 La Gaceta Diario Oficial; Managua, 18 de Febrero 2014, No 32 . Artículo 60 Cn. http://www.ineter.gob.ni/constitucion\%20politica\%20de\%20nicaragua\%20y\%20sus\%20reformas.pdf

${ }^{18}$ Versión consolidada del Tratado de Funcionamiento de la Unión Europea. https://eur-lex.europa.eu/legal-content/ES/TXT/?qid=1524848985379\&uri=CELEX:12008E/TXT

19 Alonso E. El artículo 13 del Tratado de Funcionamiento de la Unión Europea, en Favre D., GiménezCandela T. (Eds.), Animales y Derecho, Animals and the Law (Valencia 2015) 18 ss.

${ }^{20}$ Nicaragua, la nueva joya de los negocios en Centroamérica. https://www.forbes.com.mx/nicaraguala-nueva-joya-de-los-negocios-en-centroamerica/ 
Nicaragua como la nueva joya de los negocios en la región por ser un país que crecerá entre 4,5 y 5\% en el 2018 según dicha publicación ${ }^{21}$, con un crecimiento económico proyectado y similar al de los últimos 7 años, con una tasa del Producto Interior Bruto (PIB) promedio al $5,2 \%$. Esta realidad oferta al país en un escenario muy atractivo para los inversionistas y el gremio ganadero en general, dado a que si crece el país, los consumidores demandaran más alimentos.

Para tales fines el bienestar en bovinos puede brindar una mejoría en la seguridad alimentaria en lucha contra la pobreza y apoyo a las comunidades locales, igual que puede impactar en un incremento en la productividad agropecuaria, o bien puede dar mejores oportunidades de vida. A mi entender con ese fin y espíritu, fueron creadas las normas técnicas obligatorias de bienestar animal en bovinos en el año 2010, como parte del proceso de armonización para ese entonces, y se comenzó con 3 normas de bienestar ${ }^{22}$ animal $^{23}$ específicas para bovinos ${ }^{24}$, que a nuestro criterio debieron haber valorado toda la legislación vigente de derecho común, por formar parte del tejido jurídico vinculante a los animales de producción, y no solo haberse enfocado en la creación de normas técnicas obligatorias como son la:

- NTON 11-027-11: Norma Técnica Obligatoria de Bienestar de los Bovinos ${ }^{25}$

- NTON 11-035-11: Norma Técnica Obligatoria de Bienestar de los Bovinos en Establecimientos Industriales

- NTON 11-026-10: Norma Técnica Obligatoria de Sistema de Registro de Establecimientos, Identificación ${ }^{26}$ y Movilización de Ganado Bovino

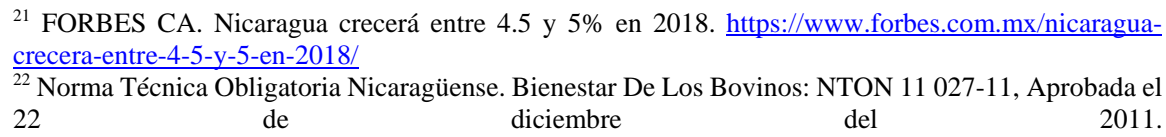
http://legislacion.asamblea.gob.ni/normaweb.nsf/(\$All)/53C3D460F2EA84EB06257A2A006E42B3? OpenDocument

${ }^{23}$ Norma Técnica Obligatoria Nicaragüense, Bienestar de los Bovinos en Establecimientos Industriales. NTON 11-035-11, Aprobado el 22 de diciembre del 2011. Publicada en la Gaceta No 110 Del 13 de $\begin{array}{lll}\text { junio } & \text { del } 2012 .\end{array}$ http://legislacion.asamblea.gob.ni/normaweb.nsf/(\$All)/8AE94097FB7EB7F306257A2A00750F4B?O penDocument

${ }^{24}$ Sistema de Registro de Establecimientos, Identificación y Movilización de Ganado Bovino. NTON 11 026-10. Aprobado el 9 de mayo del 2011 Publicada en la Gaceta No. 200 del 24 de octubre del 2011. http://legislacion.asamblea.gob.ni/normaweb.nsf/(\$All)/07EB8E994823E9230625794400502E95?Ope nDocument

${ }^{25}$ NTON: $11 \quad$ 027-11: Referencia: 11 Numeral REFERENCIAS [1] Reglamento (CE) 1/2005 del Consejo, relativo a la protección de los animales durante el transporte y las operaciones conexas y por el que se modifican las Directivas 64/432/CEE y 93/119/CE y el Reglamento

$1255 / 97$

[2] Reglamento (CE) 411/98 del Consejo, relativo a las normas complementarias sobre protección de los animales, aplicables a los vehículos de carretera utilizados para el transporte de ganado en viaje de más de ocho horas de duración. [3] Directiva 98/58 del Consejo, relativa a la protección de los animales en las explotaciones ganaderas. [4] Directiva 95/29/CE DEL CONSEJO, sobre la protección de animales durante el transporte. [5] Directiva 74/577/CEE, relativa al aturdido de los animales antes del sacrificio. [6] Reglamento (CE) 882/2004, sobre salud animal y bienestar de los animales, sobre controles oficiales efectuados para verificar el cumplimiento de la legislación en materia de piensos y alimentos y la normativa.

[7] Directiva 91/628/CEE, sobre protección de los animales durante el transporte. [8] Directiva 93/119/CEE, relativa a la protección de los animales en el momento de su sacrificio o matanza

26 NTON: $\quad$ NTON $11 \quad$ 026-10. $\quad$ Referencia 19 [1] Reglamento (CE) 178/2002 del Parlamento Europeo y Consejo, por el que se establecen los 
Desde el enfoque del Derecho Animal, no podemos pasar por alto y dejar de valorar el estatus jurídico de los animales hoy vigente, que ha estado activo desde hace más de cien años en el Código Civil. Tampoco podemos pasar por alto el efecto que ha causado el maltrato animal al estar tipificado como falta en el Código Penal nicaragüense, el cual es la única opción de sanción inmediata en el derecho común, al incumplimiento de las NTON.

Es decir, en definitiva se debió valorar más el alcance y la eficacia de las normas, puesto que no solo han dejado un enorme vacío al incumplimiento desde la administración pública, por pasarlas como desconocidas en su totalidad por la misma industria, ganaderos, y gremio académico, sino también se ha desperdiciado una gran oportunidad como valor agregado para buscar mejores soluciones para conservar e integrar la biodiversidad ante las amenazas que enfrenta la salud de los animales en el campo, como una necesidad humana. Cualquier medio o esfuerzo que se haga para conservar la naturaleza se convierte en positivo para la humanidad.

De forma más concreta quiero resaltar que la falta de protección que actualmente vive el grupo de producción bovino en Nicaragua, ante el incumplimiento de las NTON es latente y para garantizarles un bienestar óptimo no fue suficiente elaborar una norma de bienestar solo por compromisos comerciales. Esto nos puede indicar que la descripción de la ruta jurídica actual ante los estándares internacionales de bienestar animal es prácticamente inexistente bajo el esfuerzo normativo como resultado de la armonización adquirida por acuerdo internacional y regional.

Esto equivale a mencionar que los compromisos a favor del bienestar de los animales, en efecto, han quedado lejos de cumplirse y tanto el OIRSA como la OIE, si verdaderamente justifican los acuerdos adquiridos como necesarios en productividad y comercialización, entonces deberán buscar como organismos internacionales la forma de promover una asesoría más amplia a los entes que regulan la sanidad y protección de animales de Nicaragua, como solución al vacío identificado y como respuesta al estéril resultado de la norma actual.

Creo adecuado y oportuno que, tanto la OIE como el OIRSA pueden sugerir, aconsejar, o bien propiciar el inicio de revisión desde una norma con mayor eficacia como puede ser la Constitución Política de Nicaragua, para provocar un cambio real, por ser el Estado “un gestor del bien común” que debe "garantizar los intereses y las necesidades particulares, sociales, sectoriales y regionales de la nación” 27.

De lo contrario, la realidad expuesta nos seguirá enfrentando al obsoleto estatus jurídico de los animales en el Código Civil y su artículo $666^{28}$, que clasifica a los animales igual a las cosas, categoría inaceptable, inaplicable e irreal al contexto en discusión, y de

principios y los requisitos generales de la legislación alimentaria, se crea la Autoridad Europea de Seguridad Alimentaria y se fijan procedimientos relativos a la seguridad alimentaria. [2] Directiva 1992/102 del Consejo, relativa a la identificación y registro de animales. [3] Reglamento CE 1760/2000 del Parlamento Europeo y del Consejo, que establece un sistema de identificación y registro de los animales de la especie bovina y de los productos a base de carne de vacuno.

[4] Reglamento (CE) 1825/2000 de la Comisión, por el que se establecen disposiciones de aplicación del Reglamento

$1760 / 2000$.

[5] Reglamento (CE) 911/2004 de la Comisión, por el que se aplica el Reglamento 1760/2000, en lo que respecta a los dispositivos de identificación animal, los pasaportes y los registros de las explotaciones.

${ }^{27}$ Arto. 99.- El Estado es responsable de promover el desarrollo integral del país y como gestor del bien común, deberá garantizar los intereses y las necesidades particulares, sociales, sectoriales y regionales de la nación. Es responsabilidad del Estado proteger, fomentar y promover las formas de propiedad y de gestión económica y empresarial privada, estatal, cooperativa, asociativa, comunitaria, familiar, comunal y mixta para garantizar la democracia económica y social.

${ }^{28}$ Código Civil de la República de Nicaragua. Artículo Art. 666.- Los animales domésticos están sujetos a dominio que se adquiere y trasmite en la misma forma que las demás cosas. Los animales domesticados se equiparan a los domésticos, mientras conservan la costumbre de volver a la casa de su dueño. 
seguir los animales bajo ese estatus jurídico y en esa misma condición, por estar en el libro de la propiedad como bienes muebles. Entonces creo necesario analizar el estudio desde la consideración de la propiedad privada, para ampliar el rango de eficacia hasta lograr alcanzar una mayor justificación conforme a la Constitución Política de Nicaragua y su Título VI, sobre la Economía Nacional, Reforma Agraria y Finanzas Publicas, Capítulo I, referente a la Economía Nacional, y su última reforma, y primer párrafo del artículo 99, que dice:

“Arto. 99.- El Estado es responsable de promover el desarrollo integral del país y como gestor del bien común, deberá garantizar los intereses y las necesidades particulares, sociales, sectoriales y regionales de la nación. Es responsabilidad del Estado proteger, fomentar y promover las formas de propiedad y de gestión económica y empresarial privada, estatal, cooperativa, asociativa, comunitaria, familiar, comunal y mixta para garantizar la democracia económica y social.”

El artículo 99 de la Constitución Política es de inmediato la máxima herramienta jurídica en relación a la propiedad privada, y por estar los animales bajo el libro de la propiedad y por razones de productividad y comercialización ante una nación, entonces también es necesario buscar una mayor apertura hacia una respuesta a los "Derechos, Deberes y Garantías del Pueblo Nicaragüense" amparados constitucionalmente al artículo (Art. 44) de la Constitución Política de Nicaragua donde "Se garantiza el derecho de la propiedad privada de los bienes muebles e inmuebles, y de los instrumentos y medios de producción”, por ser la productividad y comercialización de animales un factor determinante en lo económico, para iniciar aquí mismo la ruta hacia un nuevo capítulo, justo y necesario, para los animales.

La ruta expuesta nos obliga a examinar de forma más amplia el actual sistema legal, valorando las debilidades y fortalezas, que a continuación resaltaré y justificaré con la intención de dar vida a un nuevo capítulo en el Código Civil a los animales en Nicaragua

\subsection{Fortalezas de la Legislación Nicaragüense a favor de los Animales Domésticos}

4.2.1. Constitución Política de Nicaragua y reforma del 2014 Ley No. 854. - Artículo 44. "Se garantiza el derecho de propiedad privada de los bienes muebles e inmuebles y de los instrumentos y medios de producción. En virtud de la función social de la propiedad, este derecho está sujeto, por causa de utilidad pública o de interés social, a las limitaciones y obligaciones que en cuanto a su ejercicio le impongan las leyes. Los bienes inmuebles mencionados en el párrafo primero pueden ser objeto de expropiación de acuerdo a la Ley, previo pago en efectivo de justa indemnización. Tratándose de la expropiación de latifundios incentivados para fines de reforma agraria la ley determinará la forma, cuantificación, plazos de pagos e intereses que se reconozcan en concepto de indemnización. Se prohíbe la confiscación de bienes. Los funcionarios que infrinjan esta disposición, responderán con sus bienes en todo tiempo por los daños inferidos. - Artículo 60. "Los nicaragüenses tienen derecho de habitar en un ambiente saludable, así como la obligación de su preservación y conservación. El bien común supremo y universal, condición para todos los demás bienes, es la madre tierra; ésta debe ser amada, cuidada y regenerada. El bien común de la Tierra y de la humanidad nos pide que entendamos la Tierra como viva y sujeta de dignidad. Pertenece comunitariamente a todos los que la habitan y al conjunto de los ecosistemas. La Tierra forma con la humanidad una única identidad compleja; es viva y se comporta como un único sistema autorregulado formado por componentes físicos, químicos, biológicos y humanos, que la hacen propicia a la producción y reproducción de la vida y que, por eso, es nuestra madre tierra y nuestro hogar común. - Debemos proteger y restaurar 
la integridad de los ecosistemas, con especial preocupación por la diversidad biológica y por todos los procesos naturales que sustentan la vida. - La nación nicaragüense debe adoptar patrones de producción y consumo que garanticen la vitalidad y la integridad de la madre tierra, la equidad social en la humanidad, el consumo responsable y solidario y el bien vivir comunitario.- El Estado de Nicaragua asume y hace suyo en esta Constitución Política el texto íntegro de la Declaración Universal del Bien Común de la Tierra y de la Humanidad”. - Artículo 98. "La función principal del Estado en la economía es lograr el desarrollo humano sostenible en el país; mejorar las condiciones de vida del pueblo y realizar una distribución cada vez más justa de la riqueza en la búsqueda del buen vivir. El Estado debe jugar un rol facilitador de la actividad productiva, creando las condiciones para que el sector privado y los trabajadores realicen su actividad económica, productiva y laboral en un marco de gobernabilidad democrática y seguridad jurídica plena, que les permita contribuir con el desarrollo económico y social del país. El Estado debe cumplir a través del impulso de políticas públicas y sociales un rol de desarrollo del sector privado, que permita mejorar la funcionalidad y eficiencia de las instituciones públicas, simplificando los tramites, reduciendo las barreras de entrada a la formalidad, avanzando en la cobertura de la seguridad social y las prestaciones sociales, y facilitando el desempeño de las empresas formales existentes. Esto se impulsará a través de un modelo de alianza del gobierno con el sector empresarial pequeño, mediano y grande, y los trabajadores, bajo el diálogo permanente en la búsqueda de consensos”. - El Artículo 99. “El Estado es responsable de promover el desarrollo integral del país y como gestor del bien común, deberá garantizar los intereses y las necesidades particulares, sociales, sectoriales y regionales de la nación. Es responsabilidad del Estado proteger, fomentar y promover las formas de propiedad y de gestión económica y empresarial privada, estatal, cooperativa, asociativa, comunitaria, familiar, comunal y mixta para garantizar la democracia económica y social.- El Estado promoverá y tutelará la cultura de la libre y sana competencia entre los agentes económicos, con la finalidad de proteger el derecho de las personas consumidoras y usuarias. Todo de conformidad con las leyes de la materia.- El ejercicio de las actividades económicas corresponde primordialmente a los particulares. Se reconoce el rol protagónico de la iniciativa privada, la cual comprende, en un sentido amplio a grandes, medianas y pequeñas empresas, microempresas, empresas cooperativas, asociativas y otras.- El Banco Central es el ente estatal regulador del sistema monetario. Los bancos estatales y otras instituciones financieras del Estado serán instrumentos financieros de fomento, inversión y desarrollo y diversificarán sus créditos con énfasis en los pequeños y medianos productores. Le corresponde al Estado garantizar su existencia y funcionamiento de manera irrenunciable. - El Estado garantiza la libertad de empresa y el establecimiento de bancos y otras instituciones financieras, privadas y estatales que se regirán conforme las leyes de la materia, los que serán supervisados, regulados y fiscalizados por la Superintendencia de Bancos y de Otras Instituciones Financieras. Las actividades de comercio exterior, seguros y reaseguros estatales y privados serán reguladas por la ley.- El Estado, con el apoyo del sector privado, cooperativo, asociativo, comunitario y mixto, en el marco de la libertad de empresa y el libre mercado, procurarán impulsar políticas públicas y privadas que estimulen un amplio acceso al financiamiento, incorporando instrumentos financieros alternativos, que profundicen y amplíen el microcrédito hacia los sectores rurales y urbanos”.

4.2.2. Código Penal. El Código Penal es relativamente nuevo comparado al código 
civil, y este ha tipificado en su Título VI capítulo VIII, de los Daños causados a los animales, y Título XV, capítulo III de los delitos Contra los Recursos Naturales, y capítulo IV, al delito de Maltrato a los Animales como faltas, sanción que no se ajusta a la realidad país, por el constante y elevado índice de maltrato a los animales en el país.

4.2.3. Ley Para La Protección y El Bienestar de los Animales Domésticos y Animales Silvestres Domesticados. Ley No. 747, Aprobada el 11 de Mayo del año 2011: pendiente de reglementar.

4.2.4. Normas Técnicas Obligatorias. Bienestar de los Bovinos en Establecimientos Industriales (NTON 11-035-11): pendiente de cumplir y aplicar.

4.2.5. Norma Técnica Obligatoria; Bienestar de los Bovinos (NTON 11027 -11): pendiente de cumplir y aplicar.

4.2.6. Norma Técnica Obligatoria: Sistema de Registro de Establecimientos, Identificación y Movilización de Ganado Bovino (NTON 11026 - 10): Se aplica de forma dispareja y solo es utilizada en la industria cárnica para bovinos, quedando el mercado informal desamparado. En cuanto a la industria láctea, no la exige.

4.3. Debilidades de la Legislación Nicaragüense en Contra de los Animales domésticos.

4.3.1. El Código Civil y la clasificación de los animales igual a las cosas en el libro II de la Propiedad, como bienes muebles. Arto. 596.- Las cosas en cuanto procuran o sirven para procurar beneficios a las personas que tienen derechos que ejercitar sobre las mismas, se llaman bienes. Arto. 597.- Los bienes consisten en cosas que jurídicamente son muebles inmuebles. Arto. 666.- Los animales domésticos están sujetos a dominio que se adquiere y trasmite en la misma forma que las demás cosas. Los animales domesticados se equiparán a los domésticos, mientras conservan la costumbre de volver a la casa de su dueño.

4.3.2. El Código Penal. La tipificación de maltrato animal como faltas ante la exclusión de gallos de pelea y corridas de toros utilizados en fiestas populares. Artículo 391. Daños físicos o maltrato a animales. "El que maltrate, someta a tratamientos crueles o se ensañe con un animal de cualquier especie, sea doméstico o no, e independientemente al uso o finalidad de los mismos, aun siendo de su propiedad, causándole daño físico por golpes, castigos o trabajos manifiestamente excesivos que lo lleven a padecer impedimentos o causen daños a su salud, estrés o la muerte, será sancionado de cincuenta a doscientos días multa o trabajo en beneficio de la comunidad de diez a veinte días por un período no menor de dos horas diarias. Quien realice espectáculos violentos entre animales, sea en lugares públicos o privados será sancionado con prisión de tres a seis meses. Si el espectáculo se realiza con ánimo de lucro, se impondrá pena de seis meses a dos años de prisión. Se exceptúa de las disposiciones anteriores los espectáculos o juegos de tradición popular, como peleas de gallos y corridas de toros”. 
Ante lo expuesto se debe propiciar una nueva reforma en materia civil y penal bajo la nueva consideración de la "Sensibilidad" de los animales, para responder al nuevo enfoque del bien supremo constitucional para promover y garantizar mejores niveles de bienestar a los bovinos con el fin de mejorar la productividad y comercialización. Así mismo, se estará garantizando a los establecimientos rurales o fincas un beneficio para restaurar la integridad de los ecosistemas con especial atención a la diversidad biológica.

\section{EL “ESTRÉS” CAUSADO POR CALOR COMO UN INDICADOR DE ALERTA PARA EL BIENESTAR ANIMAL ANTE EL CAMBIO CLIMÁTICO}

Enfrentar el "estrés 29 " como maltrato animal es un verdadero reto para el gremio ganadero, implica un desafío mayor para la nación, sobre todo en las zonas rurales donde la necesidad de veterinarios es inminente, puesto que predomina el empirismo y el rechazo a los profesionales de la salud animal. También es un reto para los expertos en Bienestar Animal y Derecho Animal, puesto que debemos coadyuvar desde la academia a todos los gremios con todas las herramientas posibles.

El fenómeno climático abre una puerta idónea para educar a toda una nación y en especial al gremio ganadero, al igual que para mejorar la conservación de la biodiversidad como un aspecto importante y sostenible del medio ambiente, determinante para el bienestar humano, donde podemos traspasar la frontera de la ignorancia hacia un verdadero cambio de cultura donde el respeto a los animales y a la madre tierra se conviertan en prioridad para el Estado.

El efecto del Cambio Climático es una preocupación social y, ante la falta de políticas públicas de Bienestar Animal, los tratos inhumanos y crueles hacia los animales seguirán siendo de todo tipo, por tanto, mitigar estas prácticas debe verse como un interés común soberano, ya que no solo afecta a los animales "per se", sino al interés común de toda una nación. Pero presionar al Estado a que sancione ${ }^{30}$ y castigue por maltrato animal no es suficiente si no hay un coparticipación estatal, de forma proactiva por ser el actor principal en materia constitucional que deberá estar a favor de los animales, reconociendo un derecho de protección de forma individual por ser inherentes a la madre tierra.

El cambio climático nos afecta a todos por igual y abrir una causa penal motivada por estrés térmico, causado por calor y altas temperaturas en bovinos, debe percibirse más bien como una gran oportunidad para implementar un amplio e incluyente programa educativo nacional, iniciando por las escuelas, para llegar hasta el último rincón de la nación. Sobre esto los ganaderos ya son conscientes de la necesidad, pero deberá promoverse y pronto.

Al respecto, la Fundación nicaragüense para el desarrollo Económico y Social ${ }^{31}$, “FUNIDES” publicó en el año 2017, en su serie de economía ambiental №2 ${ }^{32}$, el informe

\footnotetext{
${ }^{29}$ Temple D., Mainau E., Manteca X. Estrés en animales de granja. https://www.fawec.org/es/fichastecnicas/23-bienestar-general/16-estres-en-animales-de-granja

${ }^{30}$ El artículo 10 del Código Procesal Penal “CPP” y el art. 325 del CPP, describen la ruta del proceso penal para a solucionar conflictos, para restablecer la paz jurídica y la convivencia social armónica, mediante el esclarecimiento de los hechos y la determinación de las responsabilidades de los acusados. También, conforme al artículo 7 del CPP, se justifica el proceso y la aplicación de sus penas como medidas de seguridad que en justicia proceden y también en otras soluciones basada en la disposición de la acción penal, como son la mediación y acuerdos entre las partes. https://www.poderjudicial.gob.ni/pjupload/spenal/pdf/2001_ley02.pdf

${ }^{31}$ La Fundación Nicaragüense para el Desarrollo Económico y Social (FUNIDES) es una institución de pensamiento independiente que se especializa en la investigación y análisis de políticas en las áreas de desarrollo socioeconómico y reforma institucional. http://www.funides.com

${ }^{32}$ Cambio climático en el sector ganadero de Nicaragua. La Fundación Nicaragüense para el Desarrollo Económico y Social (FUNIDES) pone a su disposición el estudio sobre el cambio climático en el sector
} 
titulado, "Cambio Climático, en el sector ganadero de Nicaragua; del riesqu_climáticona la Adaptación Basada en Ecoșistemas. (AbE). El informe indica en la página 19 que en Nicaragua los estudios de vulnerabilidad ante el cambio climático se han enfocado más en el sector agrícola, con importantes aportes en el sector cafetalero (Läderach et. al, 2012) y ante el enfoque de "Adaptación basado en Ecosistema" (AbE) como método alternativo. Este no solo debe adaptarse a sistemas agrícolas, sino también al sector pecuario, que requiere de más información y análisis, "siendo el sector ganadero parte del problema de la deforestación y considerado como una importante fuente de gases de efecto invernadero que aceleran el cambio climático global, pero igual sostienen que para la ganadería, la "AbE" es una alternativa al cambio climático". El informe expone que bajo ese novedoso contexto, se utiliza la "biodiversidad ${ }^{33}$ " y los servicios ecosistémicos con el fin de aumentar la capacidad del sistema agropecuario para adaptarse a la variabilidad del cambio climático.

Entre los indicadores que FUNIDES menciona como amenazas climáticas que

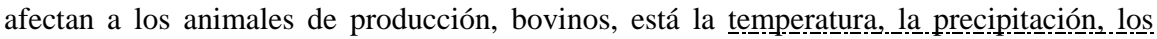

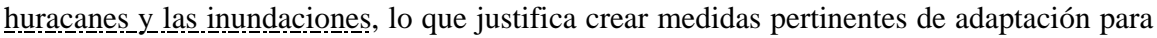
estos animales, por lo que representan las amenazas indicadas y el impacto que puede ocasionar en lo económico al sistema agropecuario del país.

Desde el enfoque especializado de la ciencia del Bienestar Animal, según el Farm Animal Welfare Education Centre ${ }^{34}$, el estrés por calor es uno de los mayores desafíos a los que se enfrentan los productores de ganado vacuno lechero en muchas regiones del mundo y se estima que hasta un $10 \%$ de la variabilidad en la producción de leche es a consecuencia de factores climáticos tales como la temperatura. Los expertos del FAWEC explican que el estrés térmico es "la sensación de calor que experimenta un animal no depende sólo de la temperatura ambiente, sino de la denominada temperatura efectiva. Ésta, a su vez, resulta de la interacción de varios factores, especialmente la temperatura ambiente, la humedad relativa, la ventilación y la radiación solar” ${ }^{35}$ y entre los efectos como indicadores también se debe valorar, además de la raza del animal y su nivel de producción, la cantidad y calidad del alimento, el estado de salud y la hidratación del animal. Sugerencia que se debe tomar en cuenta en todas las explotaciones ganaderas.

Por otro lado en la tercera edición del libro Bienestar Animal una Visión Global en Latinoamérica, se afirma que la Organización de las Naciones Unidas para la Agricultura y Alimentación (FAO), en una reunión de especialistas en el 2008, ya venía enfatizando sobre la necesidad de apoyar investigaciones en sistemas alternativos de producción enfocadas en el bienestar de los animales y en la intensificación ${ }^{36}$ de la producción animal, principalmente,

ganadero de Nicaragua. En Nicaragua alrededor del 80 por ciento de la superficie deforestada cada año es convertida en pastizales, razón por la cual ha sido criticada la actividad ganadera, además de por ser una de las actividades con mayores emisiones de gases de efecto invernadero a nivel mundial. http://www.funides.com/publicaciones/informe-y-estudios/176-cambio-climatico-en-el-sectorganadero-de-nicaragu/

${ }^{33}$ Diccionario de la Real Academia Española. Biodiversidad. http://dle.rae.es/srv/search?m=30\&w=biodiversidad

${ }^{34}$ El FAWEC (Farm Animal Welfare Education Centre. Centro de Educación en Bienestar de Animales de Producción) ha sido creado por el Servicio de Nutrición y Bienestar Animal (SNiBA) del Departamento de Ciencia Animal y de los Alimentos de la Facultad de Veterinaria de la Universitat Autònoma de Barcelona (UAB). El Centro tiene como objetivo principal la formación en bienestar de animales de producción, mediante la publicación periódica de fichas técnicas que resumen los conocimientos actuales en bienestar animal y la organización de cursos teórico-prácticos de formación sobre el bienestar del vacuno de leche y del porcino. https://www.fawec.org

${ }^{35}$ FAWEC. Temple D., Bargo F., Mainau E., Ipharraguerre I., Manteca X.. Efecto del estrés por calor en la producción de las vacas de leche: una visión práctica.

https://www.fawec.org/es/fichas-tecnicas/21-ganado-vacuno/10-efecto-del-estres-por-calor-en-laproduccion-de-las-vacas-de-leche-una-vision-practica

${ }^{36}$ FAO. Fraser D., El Bienestar y la Intensificación Animal, disponible en http://www.fao.org/3/aa0158s.pdf 
en países en vía de desarrollo, ante una visión sostenible. Según los especialistas del área, el aumento de la producción pecuaria genera una serie de cuestiones éticas, entre ellas la sustentabilidad ambiental y la garantía del acceso a los alimentos, que deben ser consideradas, en su conjunto, con la preocupación respecto al bienestar de los animales de producción ${ }^{37}$. Otro factor importante es que el bienestar ya es considerado uno de los criterios de sustentabilidad en los sistemas de producción y sobre esto algunos autores opinan que, en los sistemas intensivos, muchas veces se ignora el significado de lo que es la vida de un animal y se concibe este como una máquina que debe producir ganancias millonarias ${ }^{38}$. Ante tal actitud, se expone a los animales inclusive hasta comprometerlos físicamente hasta la muerte.

FUNIDES pudo analizar que el 94,6\% de las fincas ganaderas en los municipios seleccionados de Nicaragua se produce leche como propósito principal o como doble propósito, pero sorprendentemente se muestra que también desconocen las regulaciones o "Normas Técnicas Obligatorias de Bienestar" para garantizar la protección de los animales en los sistemas agropecuarios y ni mencionar las disposiciones establecidas en el Código Penal $^{39}$ artículo 391 y seguramente las demás ambientales.

En este aspecto, valorar el estrés en los animales puede causar un efecto muy positivo en un animal pero el hecho de valorarlo, implica varios factores. Según los expertos del FAWEC, ellos indican que "el estrés por calor reduce la concentración de proteína y grasa de la leche, inhibe la conducta de rumia y causa inmunodepresión, aumentando por lo tanto la incidencia de algunas enfermedades, el estrés por calor reduce drásticamente los índices reproductivos, ya que al disminuir la síntesis y liberación de las hormonas LH y GnRH, inhibe la ovulación y la expresión de la conducta de estro" ${ }^{40}$. Aunque el aporte técnico solo puede aplicarse de forma individual a los animales de producción, en el estudio de FUNIDES queda el interrogante sobre la reacción de las otras especies pecuarias y fauna, que de igual forma tendremos que valorar en el futuro por el efecto causado por el cambio climático.

En sus alegatos conclusivos FUNIDES, concluye que Nicaragua experimentará un calentamiento de 1,0 grados centígrados a 1,5 en las próximas décadas, relativo a los años de 1960 y 1990, bajo un escenario optimista. Igual persiste cierta incertidumbre significativa. De otra parte, expone que bajo un escenario pesimista la temperatura puede alcanzar hasta $4^{\circ} \mathrm{C}$, con fuerte tendencia de precipitaciones en las temporadas de lluvia y posibles enfrentamientos a condiciones fuertes en las temporadas de sequía en el verano. El estudio indica que las amenazas climáticas como las temperaturas elevadas, la variabilidad de precipitación, la frecuencia de los huracanes y la ocurrencia de inundaciones tiene efectos significativos en el sector ganadero del país, y sí bien cierto FUNIDES demuestra que su investigación se hizo en grupos focales con actores claves para la toma de decisiones en lo que refiere a las medidas de adaptación basadas en ecosistemas, enfocadas en amenazas climáticas específicas, también muestra que el escenario de explotación seguirá siendo igual sino se promueve un cambio de actitud en lo concerniente a las formas de explotación

\footnotetext{
${ }^{37}$ Rojas Mota D., et al., Bienestar animal, una vision global en Iberoamerica (Elsevier 2016)

${ }^{38}$ Ibídem, capitulo13

39 Código Penal, Artículo 391. Capítulo IV Maltrato A Animales, Daños físicos o maltrato a animales; El que maltrate, someta a tratamientos crueles o se ensañe con un animal de cualquier especie, sea doméstico o no, e independientemente al uso o finalidad de los mismos, aun siendo de su propiedad, causándole daño físico por golpes, castigos o trabajos manifiestamente excesivos que lo lleven a padecer impedimentos o causen daños a su salud, estrés o la muerte, será sancionado de cincuenta a doscientos días multa o trabajo en beneficio de la comunidad de diez a veinte días por un período no menor de dos horas diarias. Quien realice espectáculos violentos entre animales, sea en lugares públicos o privados será sancionado con prisión de tres a seis meses. Si el espectáculo se realiza con ánimo de lucro, se impondrá pena de seis meses a dos años de prisión. Se exceptúa de las disposiciones anteriores los espectáculos o juegos de tradición popular, como peleas de gallos y corridas de toros.

${ }_{40}$ https://www.fawec.org/es/fichas-tecnicas/21-ganado-vacuno/10-efecto-del-estres-por-calor-en-laproduccion-de-las-vacas-de-leche-una-vision-practica
} 
ganadera. Por lo que debo concluir que la necesidad de promover un cambio verdaderamente integral justificando un bien común soberano bajo una verdadera política de estado que impulse una política de bienestar animal.

\section{CONCLUSIÓN}

El país de Nicaragua es un país muy privilegiado geográficamente en la región de Centroamérica. Sus mejores testigos son las Reservas ${ }^{41}$ de Biosfera ${ }^{42}$ del Bosawas ${ }^{43}$, la Reserva de Biosfera del Sureste de Río San Juan de Nicaragua y la Reserva de Biosfera de la Isla de Ometepe; todas registradas ante la Organización de Naciones Unidas para la Educación, la Ciencia y la Cultura “UNESCO” y, hoy por hoy, se refugian en la Constitución Política del país de Nicaragua y claman el artículo número 60 de la Constitución a los nicaragüenses, por estar obligados a preservar y conservar el territorio nacional, como un bien común de la Tierra y de la humanidad. Las reservas de biosferas piden a todos los ciudadanos que entendamos la Tierra como viva y sujeta de dignidad, comunitariamente, a todos los que la habitan y al conjunto de los ecosistemas. La exclamación constitucional sobre la consideración a la madre tierra como "viva" merece, de todo, nuestra atención.

Según Giménez-Candela ${ }^{44}$, una muestra de aceptación hacia la descosificación de los animales viene mostrada en varios países del mundo, como por ejemplo Austria,

\footnotetext{
${ }^{41}$ Organización de Naciones Unidas para la Educación, la Ciencia y la Cultura (UNESCO). Reservas de Biosferas: Las reservas de biosfera son zonas compuestas por ecosistemas terrestres, marinos y costeros, reconocidas por el Programa sobre el Hombre y la Biosfera de la UNESCO. En cada una de ellas se fomentan soluciones para conciliar la conservación de la biodiversidad con su uso sostenible, el desarrollo económico, la investigación y la educación. Además, constituyen "sitios de apoyo a la ciencia al servicio de la sostenibilidad”, es decir, son zonas especialmente designadas con el objetivo de evaluar enfoques interdisciplinarios para comprender y gestionar los cambios e interacciones de los sistemas sociales y ecológicos, incluidas la prevención de conflictos y la gestión de la biodiversidad. http://www.unesco.org/new/es/naturalsciences/environment/ecological-sciences/biosphere-reserves/

${ }^{42}$ Reservas de Biosfera registradas por la UNESCO. Biosphere Reserves: Bosawas, 1997; Río San Juan, 2003; Isla de Ometepe, 2010. http://www.unesco.org/new/en/natural-sciences/environment/ecologicalsciences/biosphere-reserves/latin-america-and-the-caribbean/nicaragua

${ }^{43}$ Organización de Naciones Unidas para la Educación, la Ciencia y la Cultura (UNESCO). Mayangna, Reserva de la Biosfera de Bosawas, Nicaragua. Esta reserva de la Biosfera de Bosawas en la parte norte y central de Nicaragua es uno de los elementos centrales del denominado "Corazón del Corredor Biológico Mesoamericano". El área es reconocida por su rica biodiversidad y numerosas especies escasas o en peligro de extinción. En la región se encuentran muchas de las últimas poblaciones centroamericanas de especies como el oso hormiguero gigante, el tapir de Baird, el mono araña centroamericano, el jaguar, el águila arpía y el cocodrilo americano. En el caso de los de tapires de Baird y de monos araña, se trata de las últimas poblaciones del mundo. http://www.unesco.org/new/es/naturalsciences/priority-areas/links/biodiversity/projects/mayangna/

${ }_{44}$ Ponencia de la Dra. Teresa Giménez-Candela, "The De-objectification of Animals: Evolution or Revolution”, Congreso internacional Minding Animals, Ciudad de México, 17-24 enero 2018, https://www.youtube.com/watch?v=1GlH40XT6P4\&t=811s
} 
Alemania $^{45}$, Suiza ${ }^{46}$, Cataluña ${ }^{47}$, Francia ${ }^{48}$, Colombia $^{49}$ y Portugal ${ }^{50}$; de igual forma en la provincia de Quebec ${ }^{51}$, en Canadá. Todos ellos han comenzado este nuevo camino, el cual veo muy oportuno para Nicaragua.

Todos tenemos una cuota de responsabilidad y estamos obligados, como profesionales del derecho, a propiciar un cambio real y justo en toda la matriz jurídica vigente. La Constitución ya nos ha dado la pauta y debemos comenzar por el Código Civil.

Es hora de que los animales tengan su propio capítulo y dejen de ser vistos como cosas y, aunque sigan siendo vistos como propiedad privada, se les deberá reconocer como "seres vivos dotados de sensibilidad”, un cambio más que justo. Por tal motivo, debemos provocar nuevas políticas en la cultura, pesca, transporte, mercado interno y externo, desarrollo tecnológico, investigación, espacio e incluso el uso de animales en actos religiosos.

Si Nicaragua y toda la región Centroamericana descubren este gran beneficio, al estar a favor de la consideración de la sensibilidad de los Animales, entonces se permitirá replantear una necesidad mayor, para así involucrar a más organismos internacionales.

El reconocimiento a los animales como seres vivos dotados de sensibilidad (sentient beings) es necesario para combatir la pobreza, la seguridad alimentaria, el desarrollo sostenible en el campo, la conservación de la biodiversidad y medio ambiental, el bienestar humano y, por ende, tiene que llevarnos a la frontera idónea para combatir el cambio climático. ¡Podemos cambiar, claro que sí! Pero debemos también educarnos y aceptar a los animales como lo que son: animales.

\footnotetext{
${ }^{45}$ Giménez-Candela T., Estatuto jurídico de los animales: aspectos comparados, in Baltasar B., El Derecho de los Animales (Madrid 2015) 165-179

${ }^{46}$ Margot M., Schneider Kayasseh E., The Legal Situation Of Animals In Switzerland: Two Steps Forward, One Step Back - Many Steps To Go.

http://www.afgoetschel.com/de/downloads/legal-situation-of-animals-in-switzerland.pdf

${ }^{47}$ Ley 5/2006, de 10 de mayo, del libro quinto del Código civil de Cataluña, relativo a los derechos reales, http://noticias.juridicas.com/base datos/CCAA/ca-l5-2006.html;

El Periódico, Una mascota no es una cosa, https://www.elperiodico.com/es/sociedad/20161230/lasmascotas-son-cosas-para-la-legislacion-espanola-y-seres-vivos-para-la-catalana-5716159

${ }^{48}$ Giménez-Candela T., Una nueva Revolución Francesa : la modernización de Code civil, en dA 6/1 (2015) http://revistes.uab.cat/da/article/view/v6-n1-gimenez-candela/324

${ }^{49}$ Contreras C., Colombia: animales como seres sintientes protegidos por el derecho penal, en dA 7/1 (2016), http://revistes.uab.cat/da/article/view/v7-n1-contreras/45

${ }^{50}$ Giménez-Candela T., Reforma del Cc. de Portugal: Los animales como seres sintientes, dA 7/4 (2016), http://revistes.uab.cat/da/article/view/v7-n4-gimenez-candela/309

${ }^{51}$ The Canadian Press (12/5/2015), Quebec says animals are "sentient beings" in new protection legislation, https://ottawacitizen.com/news/politics/quebec-says-animals-are-sentient-beings-in-newprotection-legislation
} 